\title{
Financial inclusions in social networks
}

\author{
Mariia Sigova ${ }^{1, *}$, Sergey Vasiliev $^{1}$, and Oleg Kliuchnikov ${ }^{1}$ \\ ${ }^{1}$ International Banking Institute named after Anatoliy Sobchak, 191011Nevskii Ave. 60, Russia
}

\begin{abstract}
.
Research background: The study suggests that social networks can offer all the same services and products as traditional financial intermediaries. Moreover, the scope and prospects for the inclusion of financial services in the social network system are not yet fully defined and not clear. Nevertheless, the modern largest social sites are constantly developing their financial functions. Moreover, they do it in their way and often differ from the traditional financial intermediation method: firstly, social networks offer all services in a "single window" - along with other information and in the course of social communications, that is, transfer the service of network users to omnichannel, including in this concept the whole complex of socio-communication and financial interactions. The problems of the interaction of social networks with economic networks are widely studied. Quite a lot of literature is devoted to the problem of innovation in the financial sector. There are also publications that contain literature reviews, conceptual and theoretical generalizations and discussions on this issue. No less abundant electronic finance literature reviews, textbooks, numerous studies on specific issues and regional specificities of finance, as well as various web applications. Popular with researchers and the media are topics dedicated to social networks sites.

Purpose of the article in the analysis of social networks is associated with new processes, the development of new provisions, clarification of terms, the development of new hypotheses and theoretical principles.

Findings \& Value added: The challenge facing researchers can be summarized as follows: the study of information and behavioral aspects of the interaction of two types of activities - financial intermediation and social networks, leading to new processes and the formation of new organizational forms and behavior. To this end, a purposeful search is carried out for concepts, models, and tools to determine the content and prospects of these processes and new approaches are being developed and proposed.

Methods: At the first stage: (1) sociometric analysis based on graph theory, (2) concepts of interpersonal relationships, the behavior of various participants of groups and conditions for forming specific cliques in various groups, and (3) anthropological traditions, which were used to observe community members (including social networks), determine their structure, internal relations and conditions for their sustainability.
\end{abstract}

\footnotetext{
*Corresponding author: sigovamv@ibispb.ru
} 
Keywords: Financial inclusions; social networks; financial intermediaries; economic networks, network users

JEL Classification: $\mathrm{O} 1 ; \mathrm{O} 3$

\section{Introduction}

What role do online social networks play in the development of financial intermediation? How important are financial technologies for online social networks in order to provide financial services to their regulars? Is the entry of social media into the financial sector a landmark event at the turn of two decades, or is it just one of many? One view is that the entries of social networks into this area will fundamentally be transforming the financial services industry. Another is that financial inclusions in social networks is not only capable of changing social networks but also represents a new step in the construction of a modern information-financial and interconnected society, and will also become one of the key factors in lifestyle changes. Modern business, including financial intermediation and social networks, shows a high degree of interdependence [1].

According to the Center for Marketing Research at the University of Massachusetts Dartmouth (2019), the use of social networks for financial services is becoming the norm. All Fortune 500 organizations have active social media profiles (primarily on Twitter, Facebook, Instagram, YouTube, and LinkedIn). Based on a survey of financial advisors conducted at the end of 2018 , it was found that $84 \%$ of respondents used social networks (49\% in 2013); $92 \%$ of them said that social networks helped them find new customers and increase profits. In the last decade, social networks began to play an important role both in business communications (B2B) and in business relations with customers (B2C), as well as in the development of new channels and forms of customer service, new business ideas, and business models.

The transition of social networks to the provision of financial services to its users is a further development of the digitization of many socio-economic processes. Studying the entry of social networks into the provision of financial services to its users requires taking into account several different areas of knowledge, such as finance, information technology, communication technology, computer science, marketing, social relations, behavioral finance, and sociology [2].

The wider and fuller penetration of financial services into social networks is hindered by the traditional rather tight regulation of the financial sector. However, recently the flexibility of financial supervision has increased and there have been changes in the regulation of information flows.

The challenge facing researchers can be summarized as follows: the study of information and behavioral aspects of the interaction of two types of activities - financial intermediation and social networks, leading to new processes and the formation of new organizational forms and behavior. To this end, a purposeful search is carried out for concepts, models, and tools to determine the content and prospects of these processes and new approaches are being developed and proposed.

There is no definition in the literature of the term "financial inclusions in social networks", "financial insertions into social networks" or "embedding financial services in social networks". In general, switching social networks to financial services for its users is characterized by (i) financial inclusion [3] and (ii) the desire of financial intermediaries to be among consumers of financial services [4]. Financial accessibility is usually associated with solving the problem of providing financial services in regions with insufficient banking services, which is aimed at equalizing incomes and combating poverty ; The entry of financial intermediation into the social and communication environment of consumers is 
explained by the transition to a service-oriented financial economy, which is solved by providing network users with the opportunity to receive financial services as part of communication in social networks.

The problems of the interaction of social networks with economic networks are widely studied [5]. Quite a lot of literature is devoted to the problem of innovation in the financial sector. There are also publications that contain literature reviews, conceptual and theoretical generalizations and discussions on this issue. No less abundant electronic finance literature - reviews, textbooks, numerous studies on specific issues and regional specificities of finance, as well as various web applications. Popular with researchers and the media are topics dedicated to social networks sites. Issues of their development and place in the Internet space and society are widely covered. There are also various reviews of the literature devoted to social networks in general and certain aspects of their activities.

By the end of this decade, separate articles appeared on banking operations that were carried out on the basis of social media platforms [6,7]. A slightly more developed topic is the use by banks of social networks to promote their services, use the information to evaluate and attract new customers. This issue is more often considered in the context of electronic banking.

The interest of banks in social networks is dictated mainly by the tasks of the banking business. Increased competition and lower profitability push banks to penetrate new areas. In addition, social networks serve as new channels of information for expanding banking services. The study hypothesizes that the inclusion of banking services in the sphere of interests and activities of social networks will lead to a more significant event - change the place of social networks in society (including, turn them into social-financial networks), deepen and expand the scale of financialization of society [8], and make changes in a way of life.

Embedding financial services in social networks is a complex and controversial process. Any simplification, reduction or neglect of the multiplicity of factors inevitably leads to an increase in errors and a misunderstanding of the processes under study. Financial inclusions in many respects arose spontaneously. They continued the general spontaneous selforganization of social networks. Nevertheless, there were conditions for the embedding financial services in social networks [9]. They are associated with the accumulation of technological, organizational and computational developments, as well as with market, institutional and behavioral transformations that took place during the development of social networks, universal digitization and the transition to big data. The main ideas and provisions that are fundamental to the study of this topic are discussed in this section.

\section{Methods}

The use of modern ideas in the analysis of social networks is associated with new processes, the development of new provisions, clarification of terms, the development of new hypotheses and theoretical principles. Behind any technology being introduced you can always find a concept. The transition of social networks to financial inclusion, of course, was spontaneous, but it was based not only on transformations in information technology and the expansion of connections and users but also on the development of conceptual thinking. The following nine basic concepts play an important role in the development of financial services in online social networks. At the same time, these concepts indicate the main stages of development of the financial functions of social networks.

\section{- First: Sociometric approach.}

A milestone in theoretical understanding was the transition to qualitative dimensions of social relations (social acceptance, comparison of social statuses, relations between social 
structures and psychological well-being, etc.), which was associated with the use of sociometric approaches. Qualitative methods allowed discover, describe, evaluate and measure relationships within different groups .

- Second: Centrality measures and a typology of network flows.

The application of the sociometric approach to social networks prepared the basis for taking into account the location of nodes in the network, which allowed network methods and graph theory to move in the direction of establishing an indicator of centrality, which determines the most important peaks on the graph in the network. This indicator characterizes both the uniqueness and the direction of events and information on the site in a certain key topic of activity. Borgatti and Brass [10] believe that "one of the most recognizable concepts of social networks is centrality." It means that some network positions are more central, while others are more peripheral. Centrality can also be attributed to the key concept of the development of financial services in social networks. "Financial" nodes and the relations between such nodes in social networks meet the general requirements of the concept of centrality. The rise of financial functions from the periphery to the central properties are associated with the digitization and growth of data, as well as the accumulation of certain causal factors in social networks. Changing the positions of individual nodes in social networks opens up new prospects for further development, as well as the complexity of networks. From conceptual positions, the transition to multidimensional scaling of individual network nodes and assessment of the influence of nodes in complex networks is intensifying. The definition of typologies of streaming processes played a significant role in identifying and analyzing trends leading to the emergence of financial functions and their inclusion in the network mode .

- Third: Behavioral sciences, including behavioral economics and behavioral finance.

The spread of financial innovation can be analyzed in terms of the implementation of the behavioral effect and adaptation of network users to different audiences. Behavioral finance can play an important role in the study of financial functions and the new behavior of social networks. The scale of financial operations of various social networks is determined by the topology of group behavior. The transfer of financial activities to social networks changes the effectiveness market approach and leads to the spread of behavioral financial methods and tools for analyzing the behavior of social networks.

- Fourth: Two-stage communication hypothesis.

An important theoretical justification for the transition to the provision of financial services in social networks can be the two-step communication flow hypothesis, which explains how social contacts and friendly network connections influence the decisionmaking process. Despite the fact that the theory appeared in the 1940s, it received an additional impetus for development in connection with the development of web networks and especially instant messengers. Modern networks greatly simplify one-step, two-step and more complex multi-step communication models. Not only a precedent is important, but also the initiation of entering the financial sector of the main sites and messengers, as well as the transition of leaders of various groups of network sites to the use of financial services, which can lead to scaling of financial functions.

- Fifth: Agent adaptive model.

In 1997, an agent-adaptive model was proposed, revealing the effects of a convergent mechanism of social influence. It helps to explain the mechanism of the influence of the behavior of some users on the behavior of other network users, in particular, on the commission of money transactions without leaving the social site. Bandura [11] outlined the fundamental principles of the action of the human agency in the networks of sociostructural influences that are fundamental to our analysis.

Social networks act as self-organizing multi-agent systems in which various adaptation mechanisms are used. These mechanisms allow agents to spontaneously and optimally 
adapt their behavior to networking. The adaptive agent model allows explaining the behavior of decentralized agents, in particular, how the financial behavior of network users determines the behavior of the entire social network as a whole.

- Sixth: Cybersecurity issues for social and financial networks.

Problems and threats associated with the confidentiality and security of user information have become central to social and financial networks. Security issues of network use, secure transfer of financial information in networks and storage of information in the clouds, hacker attacks, occupy an important place in the general development of social and financial networks. Recently, more and more often their solution is associated with new technologies and, in particular, with technologies of distributed ledgers in social networks and finance.

Often, cybersecurity in the financial sector is associated with the stability of the financial sector, and in online social networks - with the culture of communication and various failures associated with violation of the rules of using social networks, as well as with various private manifestations of socio-cultural processes, for example, corporate culture, client culture, etc.

\section{Results}

At the turn of the two decades, recommender systems became the main channel for interaction between financial intermediaries and social networks. Financial intermediaries use them on social networks to promote their services to network users.

\subsection{Three-agent model}

The agent model is configured in such a way that it can be used to monitor agent interactions and track what happens in these interactions, that is, to explain the behavior and determine the results of agents. Modeling performed using the agent model reflects the rational behavior of the agents. In multi-agent modeling in the social sciences, a "generative" effect is allowed. It can be used to explain the mechanism of influence of financial intermediaries on consumers through recommendation systems and vice versa. In the model, you can change the behavior of agents, which leads to a change in the results. Agent behavior changes are influenced by recommendations and interactive interactions of clients with financial intermediaries.

Multi-agent modeling allows you to create a macrostructure that will reflect the real structure, for example, financial integrations in social networks. In this case, agents can generate a high level of believability of real conditions or of real actions of agents. Only in this case is the model a candidate for explaining the real relationships and behavior of agents. The assumption of a "generative" effect in agent modeling means that microspecifications can change the simulation results.

The multy-agent model of financial intermediaries, social networks and consumers, following for Brass and Borgatti [12], can be represented as a set of agents and a set of connections between them. Connections between agents are built on the basis of similarities, social relations, interactions, or flows of goods or information or their combinations (referred to as multiplicity). Moreover, the method of communication and its nature can give different results [13]. To activate a multi-agent network, it is necessary to use all agents and all connections between them.

The generating effect means the change of inference under the influence, for example, of the interaction of a financial intermediary with a client. Therefore, for each specific situation or each research question, the inference can be made. In each case, relationships are established to solve a specific problem, which can be posed by both clients and 
financial intermediaries. In such systems (for example, financial inclusion in social networks), the actions of agents are coordinated.

Coordination between agents can be carried out using various approaches, among which recommendation systems have become popular. The mechanism of agents' coordinating interactions using recommender systems will be discussed in the following sections. The recommender systems partially reflect a more familiar negotiation process since they have a special communication function that allows you to reach mutually acceptable agreements and offer the consumer a choice of services that suits his interests. Discusses negotiation processes in agent systems [14].

In the systems under consideration, a forced coordination mechanism is introduced - the recommender systems, which serve to connect financial intermediaries with consumers. They provide advice and are set up by financial intermediaries to solve their problems. Agent coordination is influenced by customer information filtering algorithms, prioritization of customer needs (queries) and the "expert search" algorithm [15], various combinations of "natural language processing" systems [16, 17], and "expert" methods ranking" [18], etc.

There are two types of relationships between financial intermediaries and consumers: direct and indirect. According to Leighton, agents are "directly" or "indirectly" related to each other in the course of participating in financial activities [19]. Direct relationships are a traditional form of relationship, which involves direct contacts of financial intermediaries with their current customers and potential customers. During the digitization of finance and the transition to online banking, these relationships are transformed. However, they retain the essence - direct contacts. The indirect nature of contacts is determined by the presence of various mediating circumstances or intermediaries that link financial intermediaries with consumers. Among such circumstances, commercial operations (e.g., buying and selling goods) hat require payments and loans are distinguished.

Social networking sites are becoming a new phenomenon in mediating financial relationships. The hypothesis is put forward that mediation is the core of the interaction of financial intermediaries and social networks. Mediation defines uniform institutional behavior, organizational convergence, and the ability to integrate specific functions.

The interaction of financial intermediaries with social networks and network users can be analyzed using a three-agent model: financial intermediary, social site, and site user (Figure 1).

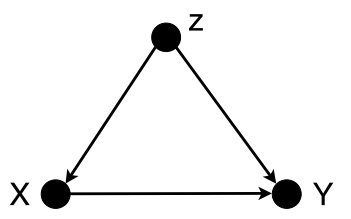

Fig. 1. The triangle of interaction between financial intermediary, social network and network user

On the proposed coordinate system, the financial intermediary acts as a predictor. He offers a financial service and can affect a network user directly (Z-Y) and/or indirectly through a social network (Z-X, X-Y).

\subsection{Conceptual model of causal}

One can propose the following conceptual model of causal relationships between a financial intermediary, a social network, and a network user (Figure 2), in which financial inclusion in social networks becomes a typical network product. 


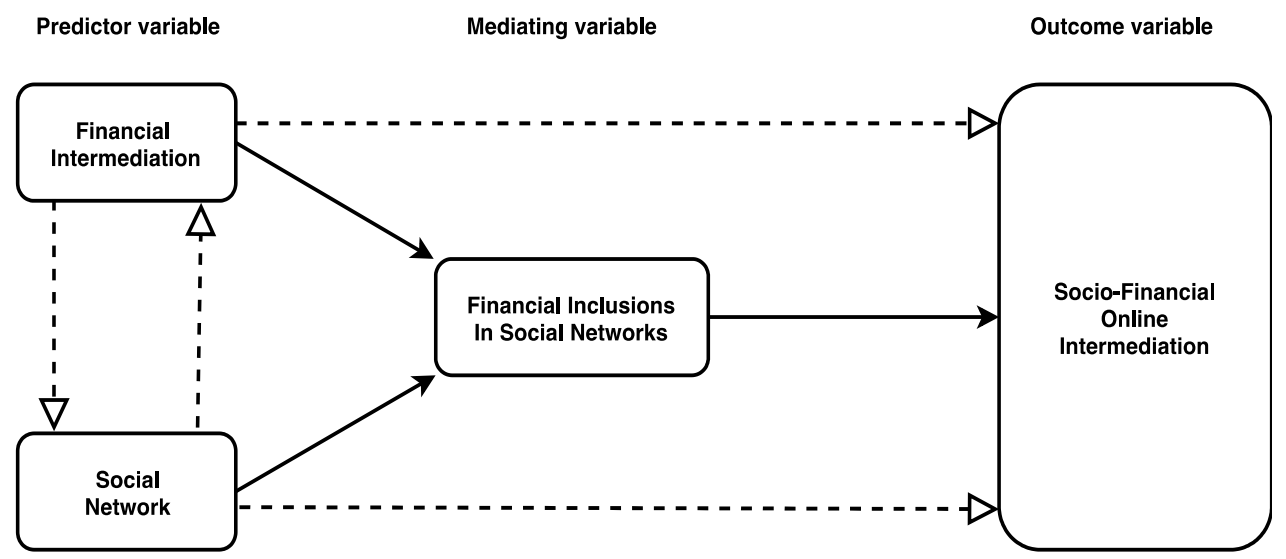

Fig. 2. Conceptual model: financial intermediary, social network, and network user

Often financial services are offered when there is a fourth element in the coordinate system (Figure 2) in question - a trade organization.

Merchandiser - the fourth agent has an impact on financial intermediation - he determines the method of payment, and together with the financial intermediary and the possibility of lending to the purchase.

In a four-agent system, the financial intermediary is a dependent variable, since its actions depend on the choice of the customer purchase method, and the client is an independent variable.

Currently, financial services through social networks are carried out mainly in connection with the servicing of trading operations performed by network users in social networks. Thus, the merging of trade channels with financial within the framework of social communications takes place. This association determines the transition to a new level of multi-channel and multi-functional ways of satisfying customer needs. Offering network users not only channels for social communication but also commercial and financial channels meet the interests of network users. It is aimed at satisfying the commercial and financial needs of customers in goods, services, payments, lending, etc. Thanks to the combination of commercial and financial services in one place, constant customer service is provided, which keeps them on the site and increases network traffic. Also, the concentration in one place of service of all the vital functions of regular visitors to the site allows you to collect various information and analyze behavior, identify needs and personalize offers. This phenomenon is presented as an omnichannel since various channels are aimed at the comprehensive satisfaction of network users' requests. Thus, it becomes possible to maintain social contacts and make purchases and payments in real-time, which saves the most valuable resource - the time of network users. Schematically, the communication lines between the three areas and the network user can be represented as follows (Figure 3). 


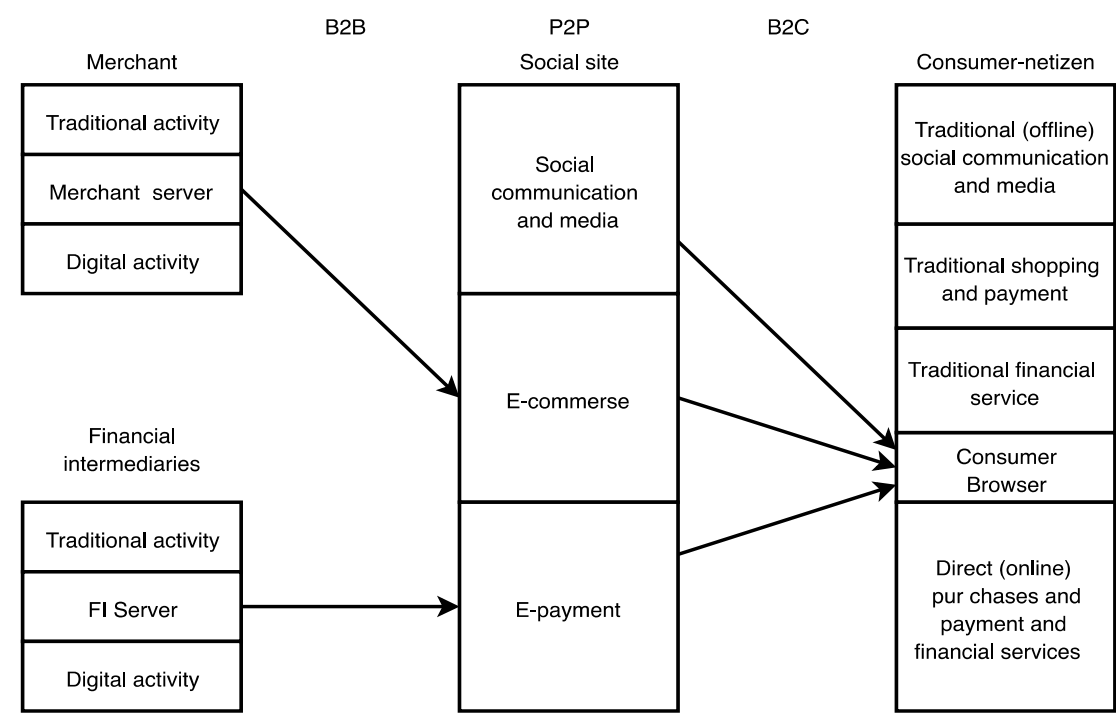

Fig. 3. A conceptual model of causal between a financial intermediary - a social network - a network user.

\section{Discussion and Conclusions}

Ideally, financial intermediation can improve performance and increase efficiency using the mediation potential of social networks, and social networks through financial inclusion can increase user satisfaction, increase traffic and fasten up users to networks. In conditions of significant acceleration of communication processes, the interaction of two types of intermediation - financial-information and social-information - can be incredibly efficient. In these circumstances, the interaction of financial intermediation and social networks can have four main effects:

- Internationalization and socialization. Specializations are changing, the system of division of labor is changing, and the system of localization of financial intermediation is being replaced by a non-spatial existence. Focusing on the internalization of norms, financial services, and financial culture, the development of universal cross-country social and financial institutions, models of behavior and actions of market agents. Financial inclusion optimizes and socializes many financial processes. They expand social connections, increase user satisfaction with networks, and create a new sociofinancial ecosystem and even lead to changes in the socio-economic construction of society.

- Individualization and self-awareness. A new social experience leading to the development of personal self-esteem. It can lead to the emergence of a new type of socio-economic behavior. The result may be the re-socialization of network users - the rejection of previous behavior from traditional forms, in particular, financial behavior, and the adoption of new ones. At the same time, with the growth of communication ties, the importance of autonomy and self-determination of individuals increases.

- Universalization of information. Financial inclusion helps to weaken and in some cases eliminate information asymmetries, reduce the amplitude of price fluctuations for financial products, equalize supply and demand, optimize calculations, save users time on financial services, etc. 
- Digitization and algorithmization. Recommender systems facilitate robotic market relations. They lead to the replacement of market regulators with computational processes.

There are many other effects, directions, and consequences of interaction, but they can all be considered as variations of the ones listed above.

The findings from the study indicated that the key result of such exposure is financial inclusions in social networks. As a result, a new form of information and financial intermediation is emerging. It is based on new technologies, an open socio-eco-system, and data. Recommender systems become one of the key conditions for the formation of financial inclusion in social networks.

\section{- Research value}

Analysis of the conceptual model for a better understanding it is always necessary to interpret the influence of the second variable (mediator) on the relationship between the predictor and the results. The second factor acts as a transitional condition for the development of two variables - financial intermediation and social networks, and it affects the final variable - the result. The study showed that social networks are beginning to mediate, firstly, financial intermediation, which increases the level of financial accessibility for users of sites, secondly, financial needs and money supply, and thirdly, monetary interactions of users of social sites. Such a conclusion is important for legislative initiatives in both financial and social spheres, as well as in the field of information technology. It can also serve as the basis for implementing a number of practical approaches in the decisionmaking process by users of social networks, heads of financial institutions and network operators. In addition, social networks act, on the one hand, as "carriers" of financial services - in cases when banking services are implemented on the margins of networks, and on the other hand, they are peculiar financial intermediaries in the framework of P2P and P2B. As a result, the company's financial coverage increases - the depth and breadth of financial coverage. In turn, social contacts often turn into financial ones. There is also a reverse movement - from financial to social.

\section{- Research limitations}

The study used qualitative data. The use of quantitative data and statistical observations may be taken in future studies. In addition, the study was cross-sectional, so future research could use a longitudinal study design. This approach will allow for a more detailed understanding of the variables being studied. In addition, in future studies, factor analysis should be taken, structural equation modeling, the use of game theory to determine the strategies and their interactions of various players, as well as the definition of the intermediary functions of social networks and financial intermediaries and the conditions for their integration.

\section{References}

1. Cheng, M.M., Liu, X.C., Wang, J., Lu, S.P., Lai, Y.K., Rosin, P.L. (2019). StructurePreserving Neural Style Transfer. IEEE Transactions on Image Processing (TIP), 29, 909-920.

2. Ardic, O.P., Heimann, M., Mylenko, N. (2011). Access to Financial Services and the Financial Inclusion Agenda around the World. A Cross-Country Analysis with a New Data Set. The World Bank.

3. Game, A. (2001). Creative ways of being. In J. R. Morss, N. Stephenson \& J. F. H. V. Rappard (Eds.). Theoretical issues in psychology: Proceedings of the International Society for Theoretical Psychology 1999 Conference (pp. 3-12). Sydney: Springer. 
4. Marinescu, D.C., Marinescu, G.M. (2012). Classical and Quantum Information Theory, in Classical and Quantum Information. Elsevier, 4, 212-344.

5. Goodfellow, I., Bengio, Y., Courville, A. (2016). Deep Learning, Deep Learning: adaptive computation and machine learning. The MIT Press.

6. Babus, A., Allen, F. (2011). Networks in Finance. Wharton Financial Institutions Center Working Paper, 8, 1-20.

7. Mobarek, A., Al-Ammary, J. H., Ezeoha, A., Sahut, J-M., Hosseini, M. H., Ghorbani, A. (2011). The E-Banking Strategies. Section 2. E-Banking and Emerging Multidisciplinary Processes. IGI Global.

8. Baruah, T.D. (2012). Effectiveness of Social Media as a tool of communication and its potential for technology enabled connections: A micro-level study. International Journal of Scientific and Research Publications, 2(5), 1-10.

9. Luo, C., Lin, Q., Liu, Y., Jin, L., Shen, C. (2020). Separating Content from Style Using Adversarial Learning for Recognizing Text in the Wild. Cornell University.

10. Fang, S., Xie, H., Chen, J., Tan, J., Zhang, Y. (2019). Learning to Draw Text in Natural Images with Conditional Adversarial Networks: Proceedings of the TwentyEighth International Joint Conference on Artificial Intelligence (IJCAI-19) (pp. 715722). Macao: Springer.

11. Cozby, P., Bates, S. (2017). Methods in Behavior Research. McGraw-Hill.

12. Hellman, Z., Peretz, R. (2020). A Survey on Entropy and Economic Behaviour. Entropy, 22(2), 157-168.

13. Ramiro, H., Gálvez, R. H., Gravano, A. (2017). Assessing the usefulness of online message board mining in automatic stock prediction systems. Journal of Computational Science, 19, 1877-7503.

14. Wasserman, S., Faust, K. (1994). Social network analyses: Methods and applications. $1^{\text {st }}$ Edition. Cambridge: Cambridge University Press.

15. Chen, X., Li, S., Li, H., Jiang, S., Song, L. (2019). Generative Adversarial User Model for Reinforcement Learning Based Recommendation System: Proceedings of the 36th International Conference on Machine Learning (ICML 2019) (pp. 1052-1061). California: Springer.

16. Hanafizadeh, P., Ravasan, A. Z., Nabavi, A., Mehrabioun, M. (2012). A Literature on the Business Impact of Social Network Sites. International Journal of Virtual Communities and Social Networking, 4 (1),1-15.

17. Salton, G., McGill, M. J. (1983). Introduction to Modern Information Retrieval. McGraw-Hill Book Co.: New York.

18. Gandomi, A., Haider, M. (2015). Beyond the hype: Big data concepts, methods, and analytics. International Journal of Information Management, 35(2), 137-144.

19. Boehmle, B., Greenwell, B. M. (2019). Hands-On Machine Learning. R. Taylor \& Francis Group. 